\title{
DETERMINACIÓN DE LAS FUERZAS CONDUCTORAS DE LA TRANSFORMACIÓN AMBIENTAL DE LA ARAUCANÍA CHILENA: EL "PAISAJE CULTURAL" COMO MARCO DE ANÁLISIS
}

\author{
DETERMINING THE DRIVING FORCES TO ENVIRONMENTAL CHANGE \\ PROCESSES OF LA ARAUCANÍA, CHILE. THE "CULTURAL LANDSCAPE" \\ AS A FRAMEWORK
}

\author{
René Montalba*, Lorena Vieli ${ }^{* *}$, Arturo Vallejos-Romero ${ }^{* * *}$, \\ Hugo Zunino ${ }^{* * * *}$ y Leonardo Vera*****
}

\begin{abstract}
El artículo propone el concepto de paisaje cultural como una perspectiva holística de análisis de procesos de transformación del paisaje. Para ello se utilizó como caso de estudio el proceso de degradación ambiental de la región de La Araucanía (Chile). Se esclarecieron las motivaciones de las actuaciones en el territorio y sus fuerzas conductoras. Estas fuerzas se relacionan a objetivos económicos externos a la población local y generaron una transformación del paisaje impactando la forma de vida de sus habitantes, quebrando el acoplamiento estructural entre población y paisaje, resultando en un paisaje cultural degradado ambientalmente.

Palabras claves: Historia ecológica, recursos naturales, acoplamiento estructural.
\end{abstract}

The notion of cultural landscape was deployed to analyze transformation processes of rural landscapes. As a case study, environmental degradation processes in La Araucania (Chile) region were analyzed. The goals of actions over the territory and their driving forces were determined. These actions were related to economic motives external to local inhabitants and produced deep transformations of the landscape and impacted the way of life of its inhabitants, breaking down the structural coupling between population and landscape, resulting in an environmentally degraded cultural landscape.

Key words: Ecological history, natural resources, structural coupling.

\section{Introducción}

En el transcurso de no más de 100 años la región de La Araucanía (ubicada en la zona centro-sur de Chile) ha pasado de tener un paisaje descrito como "selva impenetrable" (Guevara, 1898; Verniory, 2001) a otro con evidentes signos de deterioro ambiental (Universidad de Chile, 2013; Miranda et al. 2015). Las causas (e.g. Comisión Mundial sobre Medioambiente y Desarrollo [CMMD], 1991) se atribuyen a variables como la pobreza, el atraso tecnológico y el aumento de la población, que explicarían la degradación ambiental en países en desarrollo. En Chile, los discursos de organismos técnicos de desarrollo adscriben a lo expresado para explicar el deterioro ambiental en La Araucanía, en especial en los terrenos de comunidades mapuches (Morales, 2005; Montalba y Stephens, 2014). Sin desconocer la relevancia de estos factores, nuestra hipótesis es que la presión ejercida por una población local pobre y creciente sobre sus recursos naturales frágiles y escasos, no corresponde a las principales causas o motivaciones que explican el estado de conservación actual de estos recursos y el paisaje rural en La Araucanía, siendo necesario un análisis holístico que pueda entender las interpelaciones entre los sistemas naturales y socioculturales.

En el tiempo, los seres humanos han desarrollado técnicas de adaptación y uso del territorio, lo que ha dependido de las condiciones naturales, socio-económicas y culturales del territorio (Gastó, 1980). De esta forma, las actuaciones que se llevan a

\footnotetext{
* Universidad de La Frontera. Departamento de Ciencias Agronómicas y Recursos Naturales. Correo electrónico: rene.montalba@ufrontera.cl

** Universidad de La Frontera. Facultad de Ciencias Agropecuarias y Forestales. Correo electrónico: lorena.vieli@ufrontera.cl

*** Universidad de La Frontera. Núcleo de Investigación en Ciencias Sociales y Humanidades. Correo electrónico: arturo. vallejos@ufrontera.cl

**** Universidad de La Frontera. Departamento de Ciencias Sociales y Núcleo de Investigación en Ciencias sociales y Humanidades. Correo electrónico: hugo.zunino@ufrontera.cl

***** Universidad Andrés Bello. Centro de Investigación para la Sustentabilidad. Facultad de Ecología y Recursos Naturales. Correo electrónico: leonardo.vera@unab.cl
} 
cabo en el territorio no son neutras: son el producto de una cultura y una organización social que actúa sobre un nicho natural, el que posee características y dinámicas propias. El "paisaje cultural" resulta de la coevolución entre lo humano y lo natural, y lo entendemos como el producto entre las interacciones humanas y el "nicho natural", o dicho de otra forma, es lo que queda luego de la actuación del hombre en el territorio.

Ante la problemática planteada, la investigación que presentamos realiza una deconstrucción holística del proceso de generación del paisaje cultural rural en La Araucanía desde el paradigma ecológico, donde consideramos axiomáticamente que la naturaleza y el hombre se determinan recíprocamente en un contexto coevolutivo de "acoplamiento estructural" (Maturana y Mpodozis, 2000). Este acoplamiento se mantiene, o se destruye, por determinaciones mutuas que se articulan entre ambos por medio de la tecnología (Gastó, 1980). Sin embargo, aunque el hombre construye el paisaje mediante el uso de la tecnología, estas actuaciones están conducidas por motivaciones y metas donde estas "fuerzas conductoras" podrían a su vez ser diferenciadas en cinco tipos principales: económicas, políticas, tecnológicas, naturales y culturales (Bürgi, Hersperger y Schneeberger, 2004).

Por tanto, la comprensión del proceso de construcción del paisaje cultural rural de La Araucanía se lograría a partir de su contextualización en una realidad tecnológica, sociocultural y territorial determinada y de la identificación de las fuerzas directrices, su origen y del paisaje cultural resultante. De esta forma, es fundamental esclarecer si las fuerzas directrices y las causas del deterioro de los recursos naturales emergen del desarrollo (formas de actuar y medio ambiente) de una sociedad local o si es foráneo y afecta el acoplamiento estructural entre los habitantes y su entorno. No obstante lo anterior, el estudio se limita a un caso en particular (La Araucanía), donde se considera que el marco teórico como el enfoque utilizado resultan útiles para analizar y buscar soluciones a temas complejos como son los relativos al medio ambiente y desarrollo sustentable en áreas de interacción cultural y transformación del paisaje.

\section{Metodología}

Fueron utilizadas de forma complementaria una variedad de técnicas de investigación, pertenecientes a perspectivas cualitativas, cuantitativas y participativas.
Para el análisis del paisaje rural actual se utilizaron técnicas SIG y de percepción remota. Por medio de fuentes secundarias se revisaron antecedentes y estadísticas históricas relativos a los habitantes del territorio, la sociedad regional y el medio físico, colectando y sintetizando datos socioeconómicos, tecnológico-productivos y normativos, como también aquellos que dieran cuenta del estado y formas de uso de los recursos naturales. Esto fue complementado por medio de la aplicación de una entrevista semiestructurada a 69 agricultores, quienes dieron cuenta de una detallada historia oral del proceso de transformación del paisaje rural y de sus sistemas productivos. Finalmente, fueron realizadas cuatro asambleas participativas en distintas localidades de La Araucanía, donde se presentaron los resultados de la investigación, obteniendo retroalimentación de los participantes.

El estudio se desarrolló en el territorio correspondiente a la Región de La Araucanía, ubicado aproximadamente a $700 \mathrm{~km}$ al sur de Santiago de Chile (Figura 1). Esta región correspondió al último territorio mapuche autónomo (hasta 1883) (Bengoa 1991) y actualmente concentra la mayor proporción de población indígena del país (Instituto Nacional de Estadísticas [INE],2002).

\section{Transformaciones del paisaje cultural de La Araucanía}

\section{Antecedentes respecto del período prerreduccional}

A la llegada de los españoles (1550) las 3.180 .000 hectáreas de La Araucanía contaban con una población de aproximadamente 300.000 habitantes, pertenecientes principalmente al pueblo mapuche (Guevara, 1898-1902; Bengoa, 1991). En términos generales, la mayor parte del territorio se encontraba cubierto por bosques, con abundantes hongos silvestres y plantas comestibles (e.g. saprófitas, frutos, tallos, pecíolos) (Valenzuela, 1981; Smith, 1987), siendo los mapuches notables conocedores y consumidores de estos (Guevara, 1898-1902; Valenzuela, 1981). Entre los productos que recolectaban, la semilla de la conífera Araucaria araucana figura como la "harina mapuche"; si se considera la alta productividad de esta especie (hasta $400 \mathrm{~kg}$ por hectárea al año) y el valor nutricional de su semilla (232 cal/100g; 9,6\% proteína; 2,3\% lípidos), su relevancia como fuente energética y nutricional es 


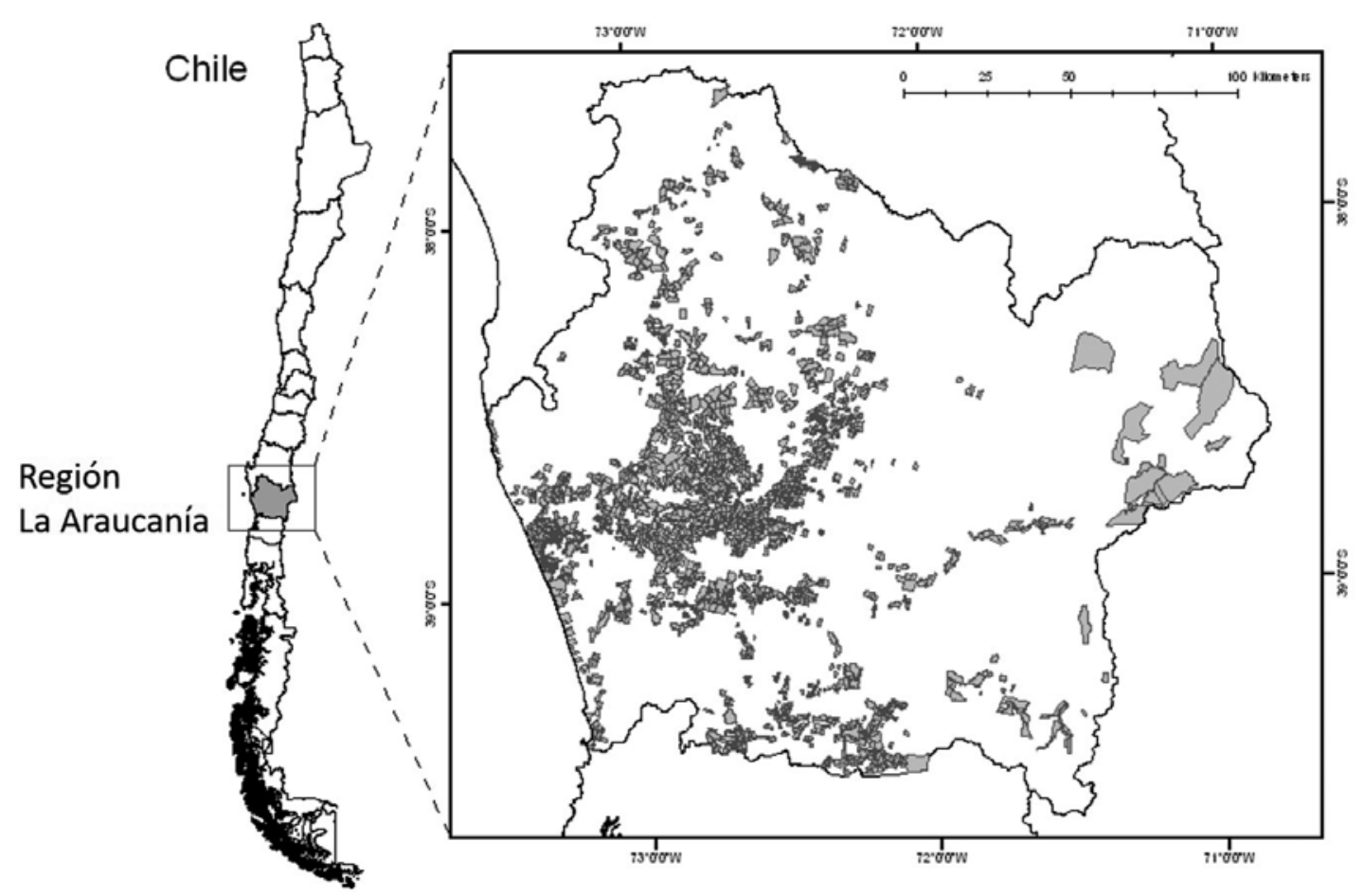

Figura 1. Mapa de ubicación de La Araucanía en Chile. Las zonas grises corresponden a títulos legales de tierras indígenas otorgados por el Estado de Chile a comunidades mapuches entre 1987 y 1929 (Fuente: Sistema Integrado de Información Indígena [SITI]. (Montalba et al., 2014).

comparable a la del trigo (Muñoz, 1984; Caro, 1995; Tacón, 1999;). Como Guevara (1989) y Bengoa (1991) mencionan, la caza también jugaba un rol importante en la alimentación de los mapuches, lo que era plausible debido a la alta abundancia y diversidad de animales existentes en los bosques (pudú, huemul, guanacos, perdices, tórtolas, torcazas, etc.) (Rozzi, Martinez, Willson y Sabag, 1997). La pesca por su parte constituía otra actividad relevante, así como también lo era la extendida ganadería de llamas para crianza doméstica y autoconsumo. Los mapuches se encontraban en un estado protoagrario, esto es, conocían la reproducción de vegetales en pequeña escala, pero no habían desarrollado aún una agricultura propiamente tal. Cultivaban papa, frijoles, maíz, quinua, ají y algunos cereales nativos menores, en muy pequeñas superficies, destinadas a suplementar los alimentos obtenidos por la caza y la recolección.

Estos antecedentes indicarían que los mapuches se desarrollaron en un ambiente rico en recursos naturales, con suficientes medios alimenticios para sustentar una alta densidad de población, obteniéndolos mediante la recolección, caza, pesca, ganadería de subsistencia y agricultura en pequeña escala. De esta forma, el bajo impacto de la actividad económico-productiva en los recursos naturales puede explicarse por los siguientes indicadores (Guevara, 1898): (i) la simpleza de las herramientas utilizadas (carecían de tracción animal, hacha, metal, ni uso del fuego para despeje de terrenos); (ii) las dimensiones y características de sus sistemas agrícolas, agroforestales y ganaderos, (iii) la abundancia de recursos naturales (alimenticios principalmente); (iv) la carencia de diferenciación o formas de acumulación en su sistema social; (v) los referentes cosmovisionales en relación con la integración del mapuche con la naturaleza y el medio que lo rodea; (vi) y el estado de conservación de recursos naturales que hasta varios siglos después presentaba La Araucanía.

La llegada de los "españoles" (1550) marca el inicio de la renombrada "Guerra de Arauco" (que duraría 300 años) y un proceso de cambio en el sistema social y económico en La Araucanía mapuche (Guevara, 1898; Bengoa, 1991). Entre los 
factores claves que impulsaron su transformación económica se encuentra la incorporación de nuevas especies animales y vegetales que se adaptaron muy bien a las condiciones de La Araucanía, adquiriendo importancia en estos cambios el caballo, el ganado vacuno, las ovejas y el trigo (Guevara, 1898; Bengoa, 1991).

El contacto con la sociedad colonial influyó en los gustos y costumbres mapuches, incorporándose varios productos provenientes del comercio. De esta forma, las sementeras adquirieron mayores dimensiones para dedicar el sobrante a la venta (intercambio), y la ganadería comienza a transformarse en la principal actividad mapuche, poseyendo un carácter mercantil. En definitiva, el sistema económico basado en la recolección de frutos, en la caza y la pesca, y en pequeñas superficies agrícolas, fue reemplazado por una economía fundamentada en el ganado vacuno, ovino y equino (Guevara, 1898; Bengoa, 1991).

Es posible deducir de este período que el uso y presión de los recursos naturales se intensificó en forma progresiva. Este proceso no estuvo relacionado a un aumento de la población, ya que esta había disminuido violentamente por enferme$\operatorname{dades}^{1}$ (Guevara, 1898), más bien se vincula a un aumento de las necesidades que trajo consigo el contacto con los españoles, y por la connotación del ganado como medio de enriquecimiento y símbolo de estatus, permitiendo la "acumulación" de recursos. Pese a esta intensificación y expansión de la actividad económica, no hay registros que indiquen problemas de degradación del bosque, agua o suelo, ya que al parecer la cosmovisión mapuche actuaba a favor de la conservación ambiental. Esta degradación sucedía en los alrededores de poblados españoles, los que rápidamente eran deforestados, abiertos a la agricultura y paulatinamente sus suelos erosionados.

Pese a la gran importancia que adquirió la crianza de ganado, no se tienen registros o relatos que indiquen que los mapuches despejaran zonas considerables de bosques para habilitar praderas o áreas de cultivo, que hubieran sobrepastoreado las praderas o producido la degradación de suelos por esta razón. Según sus referentes cosmovisionales, no todo lo que produce el $m a p u^{2}$ debe ser para el hombre, sino que el hombre es solo una parte de este mapu y se debe dejar parte de los recursos a los otros seres (naturales y sobrenaturales) que lo habitan (Quidel y Jineo, 1999; Caniullan, 2000).
Pese a lo anterior, la introducción de nuevas especies (animales como vegetales) sin duda ocasionó graves pérdidas en la agrodiversidad asociada a los mapuches, debido al reemplazo de las especies autóctonas y por las introducidas (Torrejón y Cisternas, 2002). En algunos casos se produjo la desaparición de los sistemas cultivo-crianza tradicionales y sus principales especies cultivadas (Latcham, 1936; Bullock, 1985). Así también, la expansión de la actividad ganadera debió producir alteraciones en los patrones de regeneración del bosque y de las especies asociadas a este, ya sea por daños directos, competencia con otros herbívoros, u otros mecanismos (Torrejón y Cisternas, 2002).

\section{Periodo reduccional}

En 1810, año de la independencia de Chile, el territorio mapuche gozaba de un estatus jurídico particular como consecuencia de los parlamentos realizados entre las autoridades mapuches y españolas, considerándose un reino independiente y aliado de España (Bengoa, 1991). Terminada la Guerra de la Independencia el gobierno chileno se preocupó de consolidar el país en el centro del territorio, dejando pendiente la cuestión indígena y la inclusión de La Araucanía al Estado de Chile (Vidal, 2000).

En 1848, periodo de la fiebre del oro en California y Australia, Chile comenzó a ampliar sus superficies sembradas de trigo para abastecer estos mercados (Bauer, 1970). Pese a que este mercado solo duró hasta que estos comenzaron a autoabastecerse a fines de 1850, en 1865 se inicia un período de alta demanda determinado por la Revolución Industrial y la necesidad de alimentar al naciente proletariado de base urbana, demanda que proviene de Europa Occidental, especialmente de Inglaterra (Sepúlveda, 1959). Del mismo modo, la demanda interna aumenta debido a la próspera actividad minera en Atacama, vinculada a la extracción y exportación del salitre (o nitrato de Chile), y las florecientes ciudades de Santiago y Valparaíso (Cariola y Sunkel, 1991). Las exportaciones de trigo durante la década de 1860 alcanzaron cifras aproximadas a los 1,5 millones de quintales anuales, llegando a 6,2 millones en 1874 (Bauer, 1934). Esta producción no es el resultado de la tecnificación ni de cambios estructurales en los métodos de cultivo, sino de un aumento de los terrenos cultivados, que entre 1850 y 1875 en Chile, se pasó de 120.000 ha a 450.000 ha destinadas a este cultivo (Bauer, 1970). 
De esta forma, los requerimientos de más tierras para dedicarlas al cultivo del trigo y la existencia de terrenos vírgenes en La Araucanía, fueron los factores que determinaron la anexión definitiva de estos territorios a Chile.

El 4 de febrero de 1866 los terrenos al sur del Biobío fueron declarados por ley fiscales, sin embargo dicha ocupación se pudo hacer efectiva tras la derrota militar definitiva de los mapuches. De acuerdo con la Ley de Radicación de 1883, el Estado de Chile, inspirado en California, decidió rematar, subastar o entregar esas tierras a colonos nacionales, extranjeros y miembros del ejército (Guevara, 1898-1902; Bulnes, 1985). Así también se establece para los mapuches la política de reservas indígenas, es decir, se los radica en pequeños asentamientos (reducciones) otorgados oficialmente por aproximadamente 3.000 "Títulos de Merced" (título legal de tierras indígenas) entre 1884 y 1929 (Aylwin, 2000), equivalentes a aproximadamente 470.000 ha. Estos asentamientos corresponden a $15 \%$ de la superficie de La Araucanía (Figura 1).

La derrota militar y la radicación transformó la sociedad mapuche en una comunidad de campesinos pobres, debido al despojo de los territorios de pastoreo y el fin de la actividad ganadera a gran escala. Pese a la reducción territorial y la eliminación de amplias zonas de bosques por los nuevos colonos, en gran parte del territorio mapuche las zonas boscosas residuales continuaron ocupándose para la recolección, la caza y como forraje de invierno para el escaso ganado que lograron mantener. Tal situación se expresa en los siguientes relatos de ancianos de la zona:

"Cuando éramos chicos salíamos a buscar muchas comidas naturales que salían solas por lo natural. Salíamos a buscar 'naos', 'dihueñes', nalcas, maquis, boldos, tallos de los colihues, avellanas, unas como habas y otras como arvejas silvestres, papas silvestres, rábanos y muchas otras cosas que no se sembraban. Antes también había venados libres, "luan" que estaban sueltos como pajarito, hartos pajaritos silvestres, gansos silvestres. Las quilas también eran muy importantes para los animales como forraje". F.C.N., 2011. Comunidad de Pantano

Con la llegada de los colonos y la transformación de terrenos forestales y ganaderos en cerealeros, se obtuvieron en un comienzo buenos rendimientos, indicando una gran fertilidad presente originalmente en estos sitios. Sin embargo, luego el rendimiento decrece por el agotamiento de los recursos, así como la erosión y la desertificación (Correa, 1938). La productividad de cereales fue decayendo en los sitios de altas pendientes, donde se abandona forzosamente la actividad cerealera. En los sitios de menor pendiente, menos vulnerables, se continuó con el cultivo del trigo pero se hizo necesario aumentar progresivamente los insumos productivos tecnológicos (principalmente fertilizantes) para mantener los rendimientos. Lo anterior se grafica en el relato actual de un nieto de los primeros colonos del sector cordillerano de Río Blanco:

"El trigo al comienzo nos rendía mucho. Más del 40 por uno. Independiente de si se sembraba en el faldeo o el llano. El primer potrero lo terminamos de limpiar en 1940 (plano y aluvial), y alrededor de $1955 \mathrm{el}$ trigo ya no rendía. Entonces lo empezaron a abonar con guano rojo (estiércol de aves mineralizado) y comenzó a rendir igual que al principio. En 1965 había bajado el rendimiento de nuevo y comenzaron a abonarlo con salitre. En 1970 comienzan a echarle además superfosfato triple. En 1985 había que echar el doble de abono, Aldrín y DDT pero ya no rendía y se abandonó mayoritariamente el cultivo porque ya no convenía" L.P., 2010. Valle de Río Blanco, Curacautín.

La fertilidad original de los ecosistemas que encontraron los primeros colonos fue degradada mediante la apertura del bosque y el desarrollo de la agricultura. De esta forma, se produce una simplificación del sistema, una pérdida irreversible de diversidad, y una extracción neta de la fertilidad del suelo con cada cosecha.

En la gran mayoría de los casos, la explotación de los recursos forestales de los terrenos reduccionales mapuches, así como su aprovechamiento agrícola inicial, no fueron realizados por los propios mapuches, pues no poseían herramientas ni animales de trabajo para hacerlo. Las empresas forestales y los colonos vecinos arrendaron a precios módicos el terreno y la mano de obra indígena e hicieron uso de las prácticas agrícolas que conocían, resultando 
especialmente inadecuadas para terrenos reduccionales, naturalmente frágiles por su excesiva pendiente y baja retención de nutrientes. Esto concuerda con relatos mapuches actuales:

"Contaban los antiguos que al principio preferían trabajar al día a los gringos (extranjeros) que trabajar su tierra porque no tenían bueyes para trabajar, ni herramientas como arado, carreta, yugos, lanzones y cadenas. Todas esas cosas ellos no las tenían, las tenían los gringos. La gente vivía trabajando para los gringos y recolectando las cosas naturales” D.C.H., 2011. Comunidad de Liucura.

Ante esta nueva situación los mapuches terminaron aprendiendo e imitando las técnicas y métodos agrícolas de los colonos que los rodeaban (Montalba y Stephens, 2014). Tanto estas prácticas como las herramientas que se utilizaban estaban hechas para la agricultura del valle central de Chile, de mayores extensiones y condiciones de distritos planos u ondulados menos vulnerables a la intensificación agrícola. Contrariamente, en la mayoría de los casos los mapuches estaban reducidos a pequeños terrenos con pendientes pronunciadas y alta vulnerabilidad. Respecto de la ganadería, pese a la utilización de estrategias como robar talaje a predios vecinos, buscar talaje en "los montes", o la mediería, la tecnología de manejo ganadero (de carácter extensivo) fue aplicada en pequeñas superficies (generalmente colinas) que rápidamente se sobrepastorearon y erosionaron, perdiendo buena parte del poco valor productivo que aún conservaban. Los mapuches dejaron de arrendar sus tierras, pero siguieron ocasionándole los mismos daños que les ocasionarían los colonos al imitar su tecnología y sobrepastorearlas.

No obstante lo anterior, se debe mencionar que este proceso de degradación fue mucho más intenso en los terrenos de los colonos nacionales y extranjeros. Entre 1880 y 1910, en La Araucanía se devastaron más de 500.000 hectáreas de bosque nativo y se degradaron gran parte de sus suelos (Opazo, 1910). Junto con el proceso de deforestación, el uso continuado de prácticas inadecuadas (como el barbecho) erosionó de tal forma los suelos que la mayoría de las tierras agrícolas desaparecieron de la región en un plazo no mayor a 30 años (Opazo, 1910). De esta forma, solo en las primeras décadas de control chileno del territorio, el impacto en los recursos naturales de La Araucanía (principalmente suelo, bosque, agua y diversidad) fue mucho mayor que la de toda la historia mapuche, aun considerando la época de contacto con los españoles.

Tras la crisis de los años treinta hasta los años sesenta se aplica, a nivel nacional, el modelo denominado como "nacional-desarrollismo", que buscó fundamentalmente sustituir las importaciones nacionales por producción industrial interna para desarrollar al país. La Araucanía asumió el rol de abastecedora de mano de obra a bajo precio, con lo que se da continuidad a un intenso ciclo extractivo, de degradación de los recursos naturales y de empobrecimiento de las condiciones de vida de su población.

En los años sesenta, junto con el auge de la renombrada "Revolución Verde", ocurre un cambio en la situación internacional y en el modelo político-económico de Chile. Mediante sistemas de transferencia tecnológica, créditos y subsidios estatales se produce una intencionada y profunda transformación en la gran, mediana y pequeña propiedad agrícola. Los mapuches adoptaron en mucha menor medida que agricultores de origen chileno o descendientes de "colonos" europeos los paquetes tecnológicos, inadecuados para sus condiciones y características. Aunque una buena parte de los mapuches incorporaron rápidamente las "semillas mejoradas", no disponían de capital para acceder a insumos asociados al paquete tecnológico (fertilizantes, herbicidas, hormonas, maquinaria agrícola, redes de comercialización, etc.) (Montalba y Stephens, 2014). En general cultivaron estas variedades de cultivos (de semilla mejorada) confiando en sus prácticas tradicionales de mantención de la fertilidad y en la "fuerza de la tierra", con lo que agudizaron la degradación de sus recursos naturales. Al igual que lo que ocurre en el caso de los mapuches, estudios desarrollados en distintas zonas de Latinoamérica, Asia y África, han mostrado que los primeros años tras la adopción de las nuevas "semillas mejoradas" los rendimientos se incrementan significativamente, provocando que su utilización se extienda rápidamente entre los agricultores; sin embargo, transcurridos algunos ciclos anuales bajo las mencionadas condiciones de cultivo, la situación se revierte en forma drástica, ya que estas variedades dependen de altos niveles de insumos y capital y se llega a niveles productivos bastante inferiores a los de partida (Hobbelink, 1992). 
A excepción de la incorporación de las semillas mejoradas y el uso, no generalizado, de algunos fertilizantes y pesticidas que estas imponen, los niveles de tecnología agrícola mapuche no han variado notablemente en las últimas cinco o seis décadas (fuerza de trabajo familiar o comunitaria, tracción animal, implementos agrícolas rústicos, pequeñas extensiones de cultivo). Lo que sí ha variado notablemente es el estado de conservación de los recursos naturales prediales, que se han degradado y desertificado aceleradamente. A esto se suma un aumento de la población mapuche y un vigoroso proceso de disminución y fragmentación de la superficie utilizable por ellos. De las aproximadamente 6 ha por persona asignadas en la radicación, en la actualidad se ha disminuido a solo 2 ha por persona (Aylwin, 2000; Vidal, 2000).

Otra de las aristas de los sistemas desarrollados tras la Revolución Verde es el aumento progresivo de los costos de producción y de los niveles de endeudamiento al exigir un nivel progresivamente mayor de insumos tecnológicos (fertilizantes, pesticidas y combustibles) solo para mantener la producción (Altieri, 1999). Muchos agricultores de La Araucanía que adoptaron estas prácticas y sistemas de cultivo terminaron vendiendo su mano de obra y, finalmente, vendiendo sus predios y migrando a las ciudades (Vidal, 2000).

Tras el golpe militar de 1973, la Junta Militar de Gobierno convirtió a Chile en el primer país latinoamericano en liberalizar su mercado y abrirse a la economía internacional. Se privatizaron las actividades productivas del sector público y se liberaron los precios internos y el sistema financiero. El "modelo exportador" implementado se basó en la extracción masiva de productos derivados de la minería, la agricultura, la pesca y la explotación forestal (Altieri y Rojas, 1999).

Entre otros muchos efectos, la liberalización del mercado provocó una disminución de los precios internos del trigo y el brusco aumento del precio del dólar y del petróleo elevando los precios de los insumos y con ello los costos de producción, deteriorando la rentabilidad a tal punto que la situación se tornó insostenible, tanto para la mediana como gran propiedad ganadero-cerealera. Las nuevas condiciones institucionales y macroeconómicas alentaron el desarrollo de industrias forestales basadas en monocultivos para exportación de especies exóticas de alto crecimiento. En octubre de 1974 se dicta el Decreto Ley 701 de fomento forestal, por este decreto el Estado subsidia en $75 \%$ los costos de las plantaciones forestales en terrenos calificados como preferentemente forestales. En este nuevo escenario, los endeudados agricultores vendieron grandes extensiones a las empresas forestales y $\mathrm{La}$ Araucanía fue un lugar ideal para el negocio forestal (Montalba, Carrasco y Araya, 2005).

Pese a que se podría considerar que el incremento de las superficies con especies forestales ha favorecido la conservación del medio ambiente, lo cierto es que los cultivos forestales han sido asociadas a ciertas externalidades negativas, que superan con creces los posibles beneficios ambientales que pudieran traer (Cuadro 1), las que han afectado seriamente las condiciones de vida de los mapuches y su cultura.

Junto con su alto impacto ambiental y social, la actividad forestal también ha producido un gran impacto en las frágiles economías de los sistemas de agricultura familiar, ya que junto con no incluirlos en la recepción de los beneficios económicos ofrecidos por el modelo, se ha producido un quiebre en las estrategias de subsistencia adoptadas en las distintas etapas del proceso de transformación del paisaje rural (Cuadro 2).

Por una parte, el quiebre de las estrategias de subsistencia y de otras alternativas económicas como la empleabilidad (venta de mano de obra), ha producido una mayor presión en los recursos prediales, ya que son estos los que mayormente deben dar el sustento familiar, lo que ha incrementado los niveles de degradación de los recursos naturales prediales, especialmente de las comunidades asentadas en sitios vulnerables y con bajo potencial productivo. Por otra parte, la degradación de recursos naturales incrementa los niveles de pobreza y de migración de población mapuche a las ciudades, donde pasan a formar parte de otro paisaje cultural: los periurbanos marginales.

\section{Causas de la degradación ambiental en La Araucanía y desacoplamiento estructural entre los mapuches y su paisaje}

Previo a la ocupación del Estado de Chile, los mapuches tenían una lógica desarrollada y determinada por el acoplamiento estructural entre el actor social mapuche y su territorio originario. Los principios básicos de esta lógica operaban basados en los referentes cosmovisionales, reflejados tanto en sus sistemas socioeconómicos 
Cuadro 1. Externalidades negativas asociadas a plantaciones forestales en Chile.

\begin{tabular}{ll}
\hline \multicolumn{1}{c}{ Externalidad } & \multicolumn{1}{c}{ Causa } \\
\hline Destrucción del bosque nativo & $\begin{array}{l}\text { La sustitución de bosque por plantaciones de especies exóticas es una de las principales causas } \\
\text { de destrucción del bosque nativo de Chile desde la promulgación del Decreto Ley 701 en 1974 } \\
\text { (Aguayo, Pauchard, Azócar y Parra, 2009). Solo en La Araucanía esta sustitución ha afectado } \\
\text { 30.958 hectáreas entre 1985 y 1994 (Emanuelli 1997). }\end{array}$ \\
\hline Disminución de la biodiversidad & $\begin{array}{l}\text { El establecimiento de plantaciones forestales, muchas veces reemplazando bosque nativo, } \\
\text { produce una gran reducción de la diversidad (Rozzi, Armesto y Figueroa, 1994). }\end{array}$ \\
\hline Disminución de fuentes de agua & $\begin{array}{l}\text { Las plantaciones forestales reducen las fuentes de agua (Oyarzún, Nahuelhual y Núñez, 2005; } \\
\text { superficiales y subterráneas }\end{array}$ \\
\hline Huber, Iroum y Bathurst, 2008). \\
\hline Degradación de suelos & $\begin{array}{l}\text { Tanto el establecimiento de plantaciones forestales como el manejo de estas generan degra- } \\
\text { dación de los suelos, incluyendo erosión, pérdida de nutrientes y compactación (Oyarzún y } \\
\text { Peña, 1995; Luzio, Opazo y Bascur, 1997; Oyarzún } \text { et al., 2015). }\end{array}$ \\
\hline
\end{tabular}

Fuente: elaboración propia.

Cuadro 2. Estrategias de subsistencias utilizadas por los mapuches luego de ser desplazados a las reservas indígenas, y los factores que dificultaron el éxito de estas.

\begin{tabular}{ll}
\hline \multicolumn{1}{c}{ Estrategia de subsistencia } & \multicolumn{1}{c}{ Factor que determina su ruptura } \\
\hline $\begin{array}{l}\text { Recolección de productos del } \\
\text { bosque para consumo y venta }\end{array}$ & Tala y sustitución del bosque nativo por plantaciones forestales (Emanuelli, 1997) \\
\hline $\begin{array}{l}\text { Sistemas de mediería con predios } \\
\text { vecinos }\end{array}$ & $\begin{array}{l}\text { La práctica de mediería, que consiste en que el mapuche accede a terrenos vecinos ya sea } \\
\text { para utilizar sus praderas o realizar siembras a cambio de la mitad de la producción, se ve } \\
\text { interrumpida por la venta de estos predios a empresas forestales. }\end{array}$ \\
\hline $\begin{array}{l}\text { Trabajo asalariado en predios } \\
\text { vecinos }\end{array}$ & $\begin{array}{l}\text { La venta de campos a empresas forestales y su subsecuente reforestación hace perder fuentes } \\
\text { de trabajo agrícola al solo requerir mano de obra especializada en períodos determinados } \\
\text { (Montalba, Carrasco y Araya, 2005). }\end{array}$ \\
\hline Cultivos, horticultura & $\begin{array}{l}\text { Al disminuir notoriamente la disponibilidad de agua para cultivos u hortalizas, se imposibilita } \\
\text { realizar esta actividad para la comercialización. }\end{array}$ \\
\hline Ganadería & $\begin{array}{l}\text { La dificultad de algunas zonas de conseguir agua incluso para consumo familiar, hace muy } \\
\text { difícil la mantención del ganado en verano. }\end{array}$ \\
\hline
\end{tabular}

Fuente: elaboración propia.

como en el estado de conservación de sus recursos naturales (Figura 2).

La derrota militar mapuche, y la relocalización de estos en reservas indígenas, marcan el cambio en su relación con el medio ambiente y su lógica de utilización de los recursos naturales. Desde que fueron asignados los terrenos reduccionales, los mapuches han tenido que aprender a vivir como campesinos y practicar una actividad agrosilvopastoril con una tecnología intensiva que no les es propia en territorios productivamente marginales y ecológicamente frágiles. Esto ha determinado una presión desmedida en los recursos naturales prediales, cuya resultante ha sido una degradación extrema, la pauperización de la economía mapuche y, en muchos casos, el colapso y abandono de los agroecosistemas. En esta transformación de su paisaje, los mapuches se han visto forzados sucesivamente a romper las estrategias de subsistencia que han ido desarrollando desde la radicación, por lo que aún no han podido desarrollar acciones efectivas en el dominio de su cambiante existencia. Sin embargo, en algunas zonas con características de menor vulnerabilidad y ritmo menos acelerado de cambio, se han producido interesantes adaptaciones que otorgan a los sistemas mapuche y campesino niveles considerables de sustentabilidad y resiliencia socioeconómica (Montalba et al., 2013).

No obstante lo anterior, es importante destacar que el grueso de la degradación que presenta el 


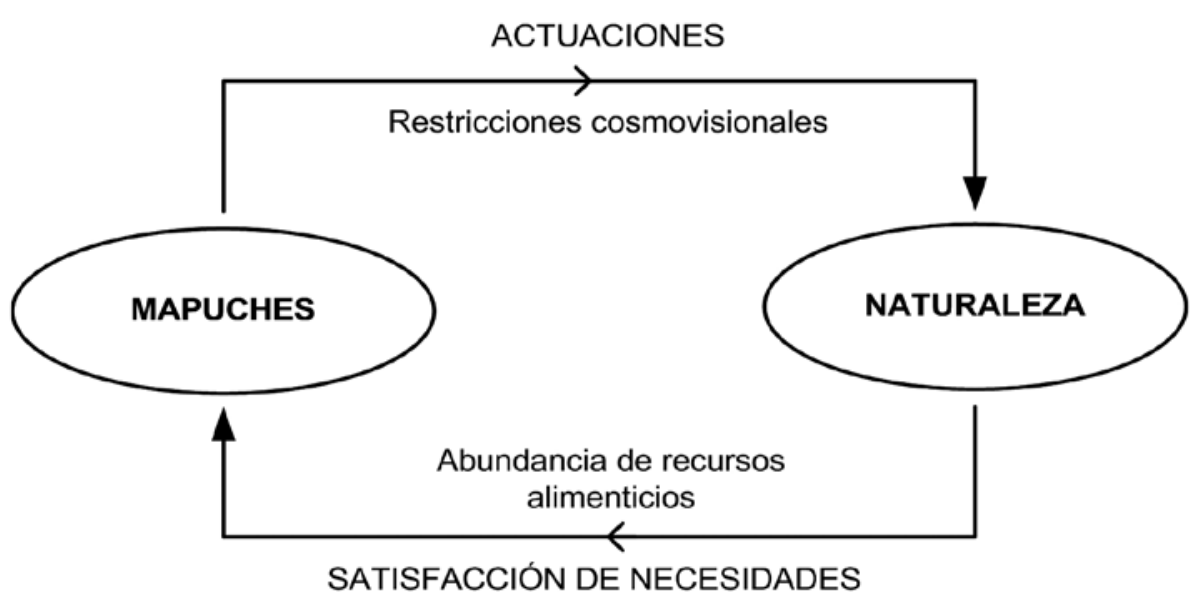

Figura 2. Esquema del acoplamiento estructural de los mapuches con su territorio. Se consideran como factores determinantes el desarrollo un territorio rico en recursos y actuaciones humanas realizadas en función a fuerzas conductoras de orden natural y social, basadas en referentes cosmovisionales.

paisaje cultural de La Araucanía no fue mediada por fuerzas directrices sociales ni culturales relativas a la presión en los recursos por parte de una población mapuche pobre y destructiva, sino que más bien a fuerzas conductoras económicas y de mercado, ya que desde un comienzo esta degradación ha obedecido a demandas de mercados externos a la región y al país. Quienes abrieron el bosque para labrar la tierra y degradaron los recursos en su etapa inicial más rica, no fueron tampoco las comunidades mapuches, sino que una pequeña fracción de la población chilena y colonos extranjeros que buscaron nuevas oportunidades de negocio. Primero fue la actividad triguera con fines de satisfacer mercados internacionales (Estados Unidos, Australia e Inglaterra, principalmente) y luego otras zonas de Chile. Tras la aplicación del modelo exportador y del colapso de los sistemas trigueros, se implanta una nueva actividad con características de mayor concentración de la propiedad y capital, con serios efectos en el medio ambiente, los recursos naturales y la calidad de vida de la población rural local: la industria forestal de exportación a gran escala.

Finalmente, es factible destacar que los períodos con menor impacto ambiental presentaron una población que en términos relativos era mucho más pobre que en períodos de mayor impacto. De esta forma, podemos considerar que la creación del actual paisaje cultural estaría en su mayoría vinculado al crecimiento de una población más rica (principalmente externa al territorio) y no al de una población pobre que busca satisfacer sus necesidades básicas.

\section{Conclusiones}

Al considerar La Araucanía como un paisaje cultural rural, y por tanto como el resultado contingente e históricamente variable de la interacción entre las fuerzas socioeconómicas y biofísicas en un territorio, fue posible analizar en forma holística el proceso de transformaciones del territorio y del estado de sus recursos naturales. De esta misma forma, la identificación de las fuerzas directrices dominantes en cada período y el origen de estos, permitió esclarecer las causas y grupos humanos vinculados a las distintas actuaciones en el territorio.

La inclusión de La Araucanía al territorio del Estado de Chile fue el hito más importante en casi 500 años de creación de su paisaje cultural, ya que esto genera una dominancia de fuerzas conductoras de orden económico y de negocio por sobre las fuerzas conductoras de tipo ecológico y social que predominaban anteriormente. Dentro de los hitos y procesos contemporáneos de mayor relevancia en la transformación del paisaje cultural de $\mathrm{La}$ Araucanía es la denominada expansión forestal y la sujeción de paisajes dominados por plantaciones de pinos y eucaliptos.

\section{Agradecimientos}

Se agradece a los agricultores y campesinos que participaron en esta investigación y a Conicyt por su financiamiento mediante el proyecto Fondecyt 1141249 y 1171144. 


\section{Referencias Citadas}

Aguayo, M., Pauchard, A., Azócar, G. \& Parra, O.

2009 "Cambio del uso del suelo en el centro sur de Chile a fines del siglo XX. Entendiendo la dinámica espacial y temporal del paisaje", en Revista Chilena de Historia Natural, $\mathrm{N}^{\circ} 82$, pp. 361-374.

Altieri, M.

1999 Agroecología, bases científicas para una agricultura sustentable. Editorial Nordan-Comunidad, Montevideo, Uruguay.

Altieri, M. \& Rojas, A.

1999 "Ecological impacts of Chile's neoliberal policies, with special emphasis on agroecosystems", en Environmental, Development and Sustainability, 1, pp. 55-72.

Aylwin, J.

2000 "Los conflictos en el territorio mapuche: antecedentes y perspectivas", en Revista Perspectivas en Política, Economía y Gestión, 3, pp. 277-300.

Bauer, A.

1934 La sociedad rural chilena desde la conquista hasta nuestros días. Editorial Andrés Bello, Santiago de Chile.

Bauer, A.

1970 "Expansión económica de una sociedad tradicional: Chile central en el siglo XIX", en Revista Historia, 9, pp. 137-235.

Bengoa, I.

1991 Historia del pueblo mapuche (siglos XIX y XX). Ediciones Sur, Santiago de Chile.

Bullock, D.

1985 "La Agricultura de los Mapuches en tiempos PreHispánicos”, en Boletín de la Sociedad de Biología de Concepción, 38, pp. 141-154.

Bulnes, G.

1985 Los mapuches y la tierra. Política y legislación chilena respecto al pueblo mapuche. Editorial PAS, Santiago de Chile.

Bürgi, M., Hersperger, A. M. \& Schneeberger, N.

2004 "Driving forces of landscape change-current and new directions", en Landscape Ecology 19, pp. 857-868.

Caniullan, V.

2000 "El mundo mapuche y su medicina", en T. Durán, E. Parada \& N. Carracsco (Eds.), Acercamientos Metodológicos hacia pueblos indígenas. Una experiencia reflexionada desde La Araucanía, Chile (pp. 123-140). Editorial LOM/ Universidad Católica de Temuco, Santiago de Chile.

Cariola, C. \& Sunkel, O.

1991 Un siglo de historia económica de Chile, 1991. Editorial Universitaria, Santiago de Chile.

Caro, $\mathrm{P}$.

1995 Producción y dispersión de semillas de Araucaria araucana (Mol.) Koch en Lonquimay, Tesis de Pregrado, Universidad de Chile, Santiago de Chile.

Comisión Mundial sobre Medioambiente y Desarrollo (CMMD) 1991 Nuestro futuro común. Alianza Editorial, Madrid.

Correa, V. L.

1938 "La ganadería", en V.L. Correa (Ed). La agricultura chilena. Editorial Nascimiento, Santiago de Chile.

Emanuelli, P.

1997 "Las cosas por su nombre: la realidad del bosque nativo de Chile", en Chile Forestal, 247, pp. 38-43.
Gastó, J.

1980 Ecología. El hombre y la transformación de la naturaleza. Editorial Universitaria, Santiago de Chile.

Guevara, T.

1868-1902 Historia de la civilización de Araucanía. Imprenta Litografía y Encuadernación Barcelona, Santiago de Chile.

Hobbelink, $\mathrm{H}$.

1992 "La diversidad biológica y la biotecnología agrícola; ¿conservación o acceso a los recursos", en Ecología Política, 4, pp. 57-72.

Huber, A., Iroumé, A. \& Bathurst, J.

2008 "Effect of Pinus radiata plantations on water balance in Chile", en Hydrological Processes, 22, pp. 142-148.

Instituto Forestal [INFOR]

2002 Estadísticas forestales 2001. Santiago de Chile.

Instituto Nacional de Estadísticas (INE) [En Linea]. Gobierno

de Chile. Santiago de Chile

2002 [Fecha de consulta]<http://www.ine.cl/canales/chile_estadistico/estadisticas_sociales_culturales/etnias/etnias.php>

Lara, A., Solari, M. E., Prieto, M., \& Peña, M. P.

2012 "Reconstrucción de la cobertura de la vegetación y uso del suelo hacia 1550 y sus cambios a 2007 en la ecorregión de los bosques valdivianos lluviosos de Chile $\left(35^{\circ}-43^{\circ} 30^{\prime}\right.$ S)", en Bosque, Vol. 33, No 1, pp. 13-23.

Latcham, R.

1936 La agricultura precolombiana en Chile y los países vecinos. Ediciones de Universidad de Chile, Santiago de Chile.

Luzio, W., Opazo, J.D, \& Bascur, C.

1997 "Variación de algunas propiedades de los suelos sometidos a tres usos diferentes", en Agricultura Técnica (Chile), Vol. 57, $\mathrm{N}^{\circ} 1$, pp. 22-28.

Maturana, H. \& Mpodozis, J.

2000 "The origin of species by means of natural drift", en Revista Chilena de Historia Natural, 73, pp. 261-310.

Miranda, A. Altamirano, A., Cayuelac, L., Pincheira, F. \& Lara 2015 "Different times, same story: Native forest loss and landscape homogenization in three physiographical areas of south-central Chile", en Applied Geography, 60, pp. 20-28.

Montalba, R. \& Stephens, N.

2014 "Ecological Change and the "Ecological Mapuche": A Historical Sketch of the Human Ecology of Chile's Araucania Region", en Human Ecology, Vol. 42, № 4, pp. 637-643.

Montalba, R., Carrasco, N. \& Araya, J.

2005 The economic and social context of monoculture tree plantations in Chile. The case of the commune of Lumaco, Araucania Region.2005. World Rainforest Movement, Montevideo, Uruguay.

Montalba, R., García, M., Altieri, M, Fonseca, F. \& Vieli, L. 2013 "Utilización del Índice Holístico de Riesgo (IRH) como medida de Resiliencia Socioecológica a condiciones de escasez de recursos hídricos, en comunidades campesinas e indígenas de La Araucanía chilena”, en Agroecología, Vol. 8, No 1, pp. 63-70.

Morales, $\mathrm{C}$.

2005 "Pobreza, desertificación y degradación de tierras", en C. Morales, S. \& Parada (Eds.), Pobreza, desertificación y degradación de los recursos naturales, Chile (pp. 25-58). Comisión Económica para América Latina y el Caribe, Santiago de Chile. 
Muñoz, R.

1984 Análisis de la productividad de semillas de Araucaria araucana en el área de Lonquimay, IX Región, Tesis de pregrado, Universidad de Chile, Santiago de Chile.

Opazo, R.

1910 Desarrollo agrícola de los territorios que constituían la antigua frontera. Imprenta Santiago, Santiago de Chile.

Oyarzún, C. Nahuelhual, L. \& Núñez, D.

2005 "Los servicios ecosistémicos del bosque templado lluvioso: producción de agua y su valoración económica", en Revista Ambiente y Desarrollo, 20-21, pp. 88-95.

Oyarzún, C.E, Hervé-Fernández, P., Huygens, D., Boeckx, P.

\& Verhoest, N.E.C.

2015 "Hydrological controls on nutrient exportation from old-growth evergreen rainforests and Eucalyptus nitens plantation in headwater catchments at southern Chile", en Open Journal of Modern Hydrology, 5, pp. 19-31.

Oyarzún, C.E. \& Peña, L.

1995 "Soil erosion and overland flow in forested areas with pine plantations at coastal mountain range, central Chile", en Hydrological Processes, 9, pp. 1099-1085.

Quidel, J. \& Jineo, F.

1999 "Las raíces para nuestro cultivo", en A. Caro, T. Duracán \& J. Tereucan (Eds.), Estilos de desarrollo para América Latina (pp. 147-158). Universidad Católica de Temuco/ Universidad Católica del Maule/Universidad de La Frontera, Temuco, Chile.

Rozzi, R., Armesto, J.J. \& Figueroa, J.

1994 "Biodiversidad y conservación de los bosques nativos de Chile: una aproximación jerárquica", en Bosque, Vol. 15, $\mathrm{N}^{\circ}$ 2, pp. 55-64.

Rozzi, R., Martínez, D., Willson, M. \& Sabag, C.

1997 "Avifauna de los bosques templados de Sudamérica", en J. Armesto, C. Villagrán \& M.K. Arroyo (Eds.), Ecología de los bosques Nativos de Chile (pp. 135-152). Editorial Universitaria, Santiago de Chile.

Sepúlveda, S.

1959 El trigo chileno en el mercado mundial. Editorial Universitaria, Santiago de Chile.
Sistema Integrado de Información Indígena (SITI)

[En línea]: Registro de Tierras Mapuche. Chile: Conadi, $<$ http://www.conadi-siti.cl>. [25 marzo 2015].

Smith, P.

1987 "Variation of water yield from catchments under grass and exotic forest, east Toago", en Journal of Hydrology, 26, pp. 175-184.

Tacón, A.

1999 Recolección de piñón y recolección de la araucaria (Araucaria araucana): un estudio de caso en la comuna de Quinquen, Tesis de Magíster, Universidad Austral de Chile, Valdivia, Chile.

Torrejón, F, \& Cisternas, M.

2002 "Alteraciones del paisaje ecológico araucano por la asimilación mapuche de la agroganadería hispanomediterránea (siglos XVI y XVII)", en Revista Chilena de Historia Natural, 75, pp. 729-736.

Universidad de Chile. Informe País: Estado del medioambiente en Chile 2012

2013 Centro de Análisis de Políticas Públicas del Instituto de Asuntos Públicos, Universidad de Chile, Santiago, Chile.

Valenzuela, $\mathrm{R}$.

1981 El Sistema Culinario Mapuche: Una Aproximación Estructural, Tesis de pregrado, Universidad de Chile, Santiago de Chile.

Verniory, G.

2001 Diez años en Araucanía. Pehuén Editores, Santiago de Chile.

Vidal, A.

2000 "Conocimiento antropológico sobre los mapuche de Chile. Efectos socioculturales y económicos de su integración forzada a la nación chilena”, en T. Durán, E. Parada \& N. Carrasco (Eds.), Acercamientos metodológicos hacia pueblos indígenas: una experiencia reflexionada desde La Araucanía, Chile (pp. 75-101). Centro de Estudios Socioculturales, Universidad Católica de Temuco, Temuco, Chile.

Wrbka T., Erb K-H., Schulz, N. B., Peterseil, J., Cahn, C. \& Haberl, $\mathrm{H}$.

2004 Linking pattern and process in cultural landscapes. An empirical study based on spatially explicit indicators", en Land Use Policy, Vol. 21, No 3, pp. 289-306.

Notas

1 El número iba desde casi 300.000 personas a la llegada de los "españoles" a cifras cercanas a las 60.000, aumentando aproximadamente a 120.000 a mediados del siglo XIX.

2 La cosmovisión mapuche considera el concepto monista de mapu, del que forma parte el hombre, la naturaleza y los seres sobrenaturales. De este concepto deriva el término mapuche, que articula el "che" (lo constitutivo de la persona) con el mapu. 
